\title{
Asporin is a stromally expressed marker associated with prostate cancer progression
}

\author{
Annie Rochette ${ }^{1}$, Nadia Boufaied ${ }^{1}$, Eleonora Scarlata ${ }^{1}$, Lucie Hamel ${ }^{1}$, Fadi Brimo ${ }^{2}$, Hayley C Whitaker ${ }^{3,4}$, \\ Antonio Ramos-Montoya ${ }^{3,5}$, David E Neal ${ }^{3,6}$, Alice Dragomir ${ }^{1}$, Armen Aprikian ${ }^{1}$, Simone Chevalier $^{1}$ \\ and Axel A Thomson ${ }^{\star, 1}$ \\ ${ }^{1}$ Department of Surgery, Division of Urology, McGill University and the Cancer Research Program of the Research Institute of \\ McGill University Health Centre, Montreal, Quebec H4A 3J1, Canada; ${ }^{2}$ Department of Pathology, Division of Urology, McGill \\ University and The McGill University Health Centre, Montreal, Quebec H4A 3J1, Canada and ${ }^{3}$ Department of Oncology, University \\ of Cambridge, Box 279, Addenbrooke's Hospital, Hills Road, Cambridge CB2 0QQ, UK
}

Background: Prostate cancer shows considerable heterogeneity in disease progression and we propose that markers expressed in tumour stroma may be reliable predictors of aggressive tumour subtypes.

Methods: We have used Kaplan-Meier, univariate and multivariate analysis to correlate the expression of Asporin (ASPN) mRNA and protein with prostate cancer progression in independent cohorts. We used immunohistochemistry and $\mathrm{H}$ scoring to document stromal localisation of ASPN in a tissue microarray and mouse prostate cancer model, and correlated expression with reactive stroma, defined using Masson Trichrome staining. We used cell cultures of primary prostate cancer fibroblasts treated with serum-free conditioned media from prostate cancer cell lines to examine regulation of ASPN mRNA in tumour stromal cells.

Results: We observed increased expression of ASPN mRNA in a data set derived from benign vs tumour microdissected tissue, and a correlation with biochemical recurrence using Kaplan-Meier and Cox proportional hazard analysis. ASPN protein localised to tumour stroma and elevated expression of ASPN was correlated with decreased time to biochemical recurrence, in a cohort of 326 patients with a median follow up of 9.6 years. Univariate and multivariate analysis demonstrated that ASPN was correlated with progression, as were Gleason score, and clinical stage. Additionally, ASPN expression correlated with the presence of reactive stroma, suggesting that it may be a stromal marker expressed in response to the presence of tumour cells and particularly with aggressive tumour subtypes. We observed expression of ASPN in the stroma of tumours induced by p53 inhibition in a mouse model of prostate cancer, and correlation with neuroendocrine marker expression. Finally, we demonstrated that ASPN transcript expression in normal and cancer fibroblasts was regulated by conditioned media derived from the PC3, but not LNCaP, prostate cancer cell lines.

Conclusions: Our results suggest that ASPN is a stromally expressed biomarker that correlates with disease progression, and is observed in reactive stroma. ASPN expression in stroma may be part of a stromal response to aggressive tumour subtypes.

Prostate cancer (PCa) is highly prevalent and represents $24 \%$ of cancer diagnoses in Canadian men; in 2016, about 27000 Canadian men will be diagnosed and 4100 will die from the disease. The prevalence of PCa increases with age and there is a large pool of indolent PCa which is best managed with active surveillance. However, there is a limited ability to distinguish lethal from indolent disease, resulting in many patients opting for intervention and risking side effects and over-treatment. To address this, several genomic, transcript and immunohistochemical biomarkers have been assessed for their abilities to identify 
progressive tumour subtypes and aid treatment choice between intervention and surveillance (Martin, 2016). There is considerable inter- and intra- tumour heterogeneity which limits the efficacy of predictive tests and most focus upon the ability to detect tumour subclones associated with progression, which may be present at low frequency in biopsy samples. We have focussed upon the possibility that tumour stroma may exhibit markers expressed in response to aggressive tumour subclones, which may help to distinguish these from indolent tumour subtypes.

Cancer associated fibroblasts (CAFs) play a key role in tumourigenesis by regulating tumour cell proliferation and invasion, via mechanisms including growth factors, cytokines, ECM stiffness, and immune cell trafficking (Olumi et al, 1999). The histology of tumour stroma is predictive of disease progression (Jia et al, 2011; Planche et al, 2011; Jia et al, 2012; Tian et al, 2015), and particularly the presence of reactive stroma and collagen deposition (Ayala et al, 2003; Yanagisawa et al, 2007). There are several markers of CAFs such as FSP1, smooth muscle actin, CD90, PDGFR, and FAP, which show partially overlapping expression, though these are not predictive of disease progression. Gene profiling studies have analysed prostate tumour stroma and CAFs, with focus upon reactive stroma (Dakhova et al, 2009), CD90 positive stroma (Pascal et al, 2009), and primary CAFs (Orr et al, 2012) and all noted expression of ASPN mRNA in tumour associated prostate stroma.

Asporin was identified among androgen responsive genes in murine prostate development (Schaeffer et al, 2008), and using immunohistochemistry we observed a localised expression pattern of ASPN in subsets of human fetal prostate mesenchyme located adjacent to developing prostatic buds (Orr et al, 2012). In tumour stroma, ASPN was observed surrounding tumour cells suggesting a possible role in progression. Interestingly, ASPN was expressed in stroma with a partially overlapping pattern with stanniocalcin 1 (STC1), another molecule implicated in CAF regulation of tumourigenesis (Pena et al, 2013). ASPN levels are elevated in the blood of men with advanced prostate adenocarcinoma (Klee et al, 2012), and it is detectable in exosomes derived from saliva of tumour-bearing mice (Lau et al, 2013).

Asporin, also known as periodontal ligament associated protein (PLAP1) is a secreted small leucine rich proteoglycan with known roles in ligament regulation and chondrogenesis via BMP and TGBbeta pathways. It regulates BMP2, TGF $\beta$ and FGF2 activity through direct and indirect interactions (Kizawa et al, 2005; Yamada et al, 2007; Tomoeda et al, 2008; Awata et al, 2015) and also binds type II collagen (Kou et al, 2010). ASPN has been shown to play a role in invasion in breast cancer cells (Simkova et al, 2016) and control the invasion of tumour cells and CAFs in scirrhous type gastric cancer (Satoyoshi et al, 2014), both in vitro and in vivo via the CD44-Rac1 pathway (Satoyoshi et al, 2014). In prostate cancer, varying aspartic acid repeat length in the $\mathrm{N}$-terminus of ASPN is associated with prostate cancer progression (Hurley et al, 2015). In contrast, in breast cancer, a lack of ASPN expression in the stroma of triple negative cases is associated with poor prognosis (Maris et al, 2015).

We have examined the role of ASPN in prostate cancer progression, at the mRNA and protein level, in data sets with clinical outcome. The association of ASPN with reactive stroma was investigated, as well as expression of ASPN in the stroma of a murine prostate cancer model. Finally, effects of tumour cell factors upon ASPN expression in stromal cells were studied in vitro.

\section{MATERIALS AND METHODS}

Human tissue samples. This study was approved by the Ethics Board of the McGill University Health Centre. Written informed consent was obtained from all the participants. We used a tissue microarray (TMA) composed of 5 FFPE blocks containing samples of 326 prostatectomy specimens of hormone naive patients from 1996 to 2007 . Histopathologic review of all cases was performed by one genitourinary pathologist (FB) using the modified Gleason grading system (2015). Briefly, the benign and malignant regions of all cases were identified and two $1.0 \mathrm{~mm}$ cores were extracted from the cancer area of the dominant nodule. One core of benign tissue away from cancer was included for $20 \%$ of the cases. The TMA was constructed by using a Beecher MT1 manual arrayer (Beecher Instruments, Silver Spring, MD, USA). A commercial TMA was purchased from USBioMAx.

Mouse TP53 prostate cancer model. $\mathrm{PB}-\mathrm{Cre} / \mathrm{p} 53^{\text {loxP/loxP }} \mathrm{Rb}^{\text {loxP/loxP }}$ mice develop low-grade PIN in the four lobes, but the aggressive, castrate resistant metastatic tumours that develop in these mice arise from the proximal region of the ducts near the urethra (Zhou et al, 2006, 2007). Tissue was collected from males between 5.5 and 7.7 months of age. Mice were maintained in the Cancer Research UK Cambridge Institute Animal Facility. All animal procedures were carried out in accordance with University of Cambridge and Cancer Research UK guidelines under UK Home Office project license 80/2435.

Statistical analysis. All statistical analyses were performed using R (Team, R.D.C., 2011). Survival curves and Cox proportional hazard were conducted with survival package (Therneau, 2015), survminer (Kassambara, 2016) and survcomp (Schroder et al, 2011); the $P$-values were computed using the log-rank test.

Immunohistochemistry. Immunostaining of ASPN on TMA was performed using a rabbit anti-ASPN antibody (Sigma, HPA008435) diluted 1:80. TMAs were de-paraffinised in xylene and hydrated in ethanol and rinse under water. Endogenous peroxidase activity was blocked for $30 \mathrm{~min}$ with $3 \% \mathrm{H}_{2} \mathrm{O}_{2}$. After a $3 \mathrm{~h}$ block with IHC Select (Millipore, Billeria, MA, USA), the primary antibody was used overnight. Histofine Simple Stain MAX PO multi (Nichirei BioSciences, Inc., Tokyo, Japan) was used as secondary antibody and chromogenic detection was carried out using Simple Stain AEC Solution (Nichirei BioSciences, Inc.). For immunostaining of null mouse tissues, slides were pressure cooked in $10 \mathrm{mM}$ citric acid, pH6.0 (Poly Scientific R\&D), for $5 \mathrm{~min}$ before staining. IHC was performed using a rabbit anti-Aspn antibody (Genetex, Irvine, CA, USA, GTX104790) diluted 1:1600 and anti-synaptophysin (BD Biosciences, San Jose, CA, USA, BD611880) diluted 1:100. Images were taken with an Aperio Slide Scanner (Leica, Wetzlar, Germany).

IHC data analysis. Asporin staining was assessed using the staining $H$-score method (Bosman et al, 1992). $H$-score was obtained by computing staining intensity $(0-3)$ and the proportion of stroma cells stained for each intensity $(0-100)$ to give an $H$-score between 0 and 300 .

Reactive stroma was defined according to published criteria (Yanagisawa et al, 2007) after staining with Masson's Trichrome.

Cell lines. Normal adults prostate stromal cells, PrSC (Lonza), primary culture of embryonic prostate fibroblast (EPF) (Orr et al, 2011), cancer-associated fibroblasts (CAF) (Orr et al, 2011), WPMY-AR (Tanner et al, 2011), BHPrS (Franco et al, 2011), PC-3 (ATCC), and LNCaP (ATCC) were grown in Dulbecco's modified Eagle medium GlutaMAX (Gibco, Waltham, MA, USA) with $10 \%$ fetal bovine serum, without antibiotics.

For conditioned media, PC-3 and LNCaP cells $\left(6 \times 10^{6}\right)$ were plated in $150 \mathrm{~cm}^{2}$ flasks and grown overnight. Cells were washed with PBS and medium was replaced with serum free DMEM GlutaMAX and incubated for $24 \mathrm{~h}$. The medium was then collected, centrifuged to remove cell debris and passed through a $0.22 \mu \mathrm{m}$ filter. Stromal cells were plated in $60 \mathrm{~mm}$ dishes and grown overnight. Cells were washed twice in serum free DMEM and conditioned media was 
added for $24 \mathrm{~h}$. RNA was extracted and cell proliferation was assessed using CellTiter-Glo (Promega, Fichburg, WI, USA).

RNA extraction and quantitative real-time PCR. Total RNA was extracted using the EZ-10 Spin Column Total RNA Minipreps (BioBasic) following manufacturer's instructions. Complementary DNA was performed using High Capacity cDNA Reverse Transcription (ABI) and qRT-PCR was performed on ABI 7500 Fast machine using SYBR Select Master Mix. Transcript abundance was normalised to TBP expression. Primers used for TBP were $5^{\prime}$-GGGGAGCTGTGATGTGAAGT- $3^{\prime}$ and $5^{\prime}$-ACCAGGAAATAACTCTGGCTCAT- $3^{\prime}$ and for ASPN were $5^{\prime}$-TTGAAGGGGTGA-

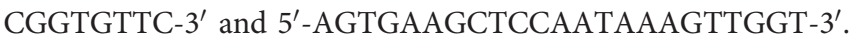

\section{RESULTS}

Expression of ASPN mRNA in patient cohorts. Increased expression of ASPN mRNA has previously been observed in cancer- associated fibroblasts by gene profiling studies (Dakhova et al, 2009; Orr et al, 2012; Hurley et al, 2015), and some patient cohorts (Hurley et al, 2015). We extended these observations using a cohort with patient follow up to investigate correlation of ASPN mRNA with biochemical recurrence (BCR) and other clinical criteria. The association between ASPN expression, disease progression, and recurrence was investigated using a recently published prostate tumour profiling data (Mortensen et al, 2015) derived from 36 microdissected tumour tissues with 14 patient matched benign tissues. In the Mortensen data set tumour samples showed a 1.3-fold increase of ASPN levels compared to patient matched benign tissue (Figure 1A). Kaplan-Meier survival analysis showed that patients with elevated levels of ASPN had a higher rate of biochemical recurrence $P=0.0011$ (Figure 1B). In univariate and multivariate Cox hazards ratio analysis (Table 1A) ASPN showed a significant association with biochemical recurrence $(\mathrm{HR}=2.16 ; P=0.003$; HR 2.23; $P=0.009)$. Other parameters such as Gleason score, stage, and positive margins were also associated with BCR (Table 1A). Overall, these data suggest that ASPN mRNA levels correlated with increased probability of disease progression in the Mortensen data set similar to that observed in other data sets (Hurley et al, 2015).
Immunohistochemical analysis of ASPN in prostatectomy tissues. We next examined the distribution of ASPN protein using immunohistochemistry in order to define distribution within tumour stroma of patient samples, and to examine association of ASPN with clinical parameters. We performed immunohistochemistry using a TMA of prostate cancer tissue from a cohort of 326 patients who underwent radical prostatectomy. Clinico-pathological characteristics of the patient cohort are listed in Supplementary Table S1. Median age was 61 (range 43-73), median follow up was 9.6 years (range $0-20.8$ ) and the percentage of patients showing BCR was $25 \%$ (81). Asporin was localised using an antibody validated in several independent studies (www.proteinatlas.org, (Hurley et al, 2015; Maris et al, 2015; Satoyoshi et al, 2014; Simkova et al, 2016). After immunohistochemistry, ASPN staining was assessed using the $H$-score method which involved computing staining intensity ( 0 to 3 ) and the proportion of stroma stained ( 0 $100)$ to give an $H$-score between 0 and 300 . Most patient samples consisted of duplicate cores and each core was examined by two individuals and $H$-scores determined. The $H$-scores from duplicate spots were averaged prior to analysis. Asporin staining was evident in tumour stroma with a broad range of staining intensity and extent, examples of $H$-score $0,100,200$, and 300 are shown (Figure 2A, high magnification shown in Supplementary Figure 1). 240 patients had an $H$-score between $0-100,46$ patients between 101-150, 23 patients between 151-200, 7 patients between 201-250 and 4 patients higher than 251 . $H$-score of benign samples was less than 30 for $91 \%$ of the samples, confirming that ASPN was low or absent in normal prostate tissue, and consistent with lower ASPN mRNA levels in benign tissue (Figure 1A).

We observed a correlation between increased $H$-score and biochemical recurrence; patients with an $H$-score above 150 showed a $41 \%$ biochemical recurrence rate compared to $22 \%$ among the remainder $(P=0.0077$; Figure $2 \mathrm{~B})$. Univariate and multivariate analysis of ASPN $H$-score in the McGill cohort showed hazards ratios of $2.16(P=0.009)$ and $1.77(P=0.062)$ for BCR (Table 1B), supporting the association of ASPN with progression. We next examined the expression of ASPN within patients with Gleason score of 7; this showed that those patients positive for ASPN showed more rapid BCR than those who did not $(P=0.0012$; Figure 2C). Univariate and multivariate analysis showed hazards ratios of $2.52(P=0.002)$ and $2.30(P=0.016)$ for ASPN and BCR within the Gleason 7 subgroup (Table 1C).
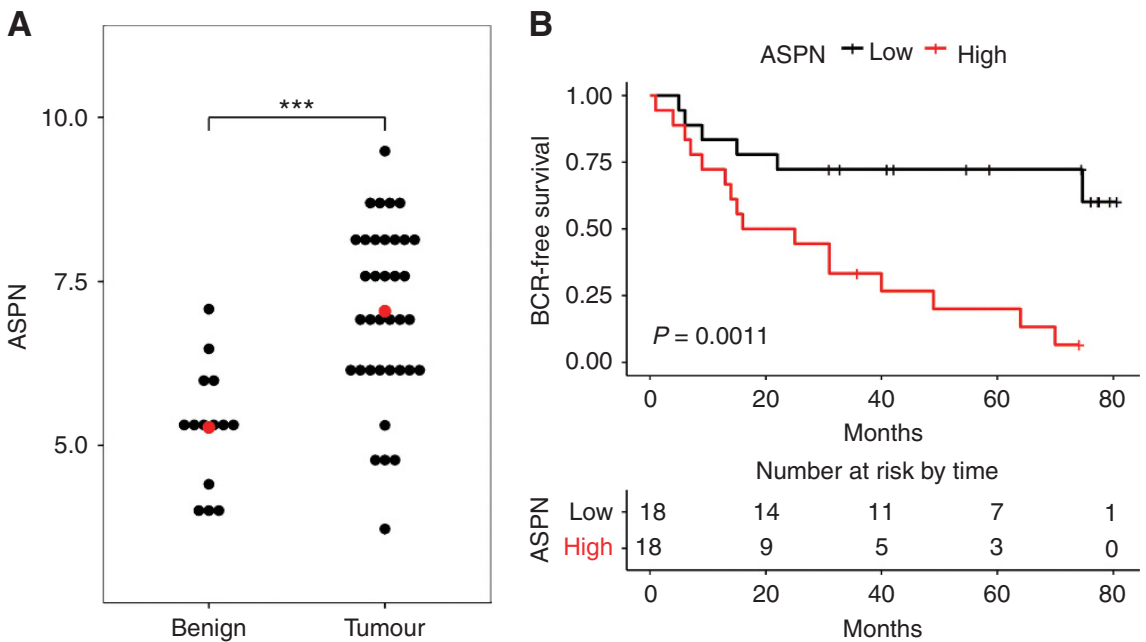

Figure 1. Correlation of ASPN mRNA with BCR in the prostate cancer patient cohort described by Mortensen et al (2015). (A) A significant increase in ASPN transcripts was observed in tumour vs patient matched benign samples; mean ASPN mRNA levels of 7.0 in tumour samples vs 5.3 in benign samples ( $t$-test, $P<0.001)$. (B) Kaplan-Meier analysis correlated higher ASPN transcript levels with shorter time to biochemical recurrence $(P=0.0011)$ in the Mortensen et al (2015) data set. The lower panel shows the number of patients at risk of BCR during 80 months of follow up. 
Table 1A. Hazards ratios of ASPN and BCR; for ASPN mRNA in Mortensen et al, and ASPN H-score in the McGill TMA. Cox analysis of ASPN mRNA and BCR in the Mortensen et al data set

\begin{tabular}{|c|c|c|c|c|c|c|c|}
\hline & \multicolumn{4}{|c|}{ Univariate analysis } & \multicolumn{3}{|c|}{ Multivariate analysis } \\
\hline & $N(n)$ & HR & $95 \% \mathrm{Cl}$ & $P$-value & HR & $95 \% \mathrm{Cl}$ & $P$-value \\
\hline ASPN & $36(22)$ & 2.16 & $1.30-3.59$ & 0.003 & 2.23 & $1.32-4.66$ & 0.009 \\
\hline Gleason score & $36(22)$ & 1.75 & $1.19-2.58$ & 0.005 & 1.44 & $0.77-2.60$ & 0.228 \\
\hline PSA & $36(22)$ & 1.07 & $1.03-1.12$ & 0.001 & 1.08 & $1.02-1.14$ & 0.005 \\
\hline Stage & $33(22)$ & 4.21 & $1.60-11.08$ & 0.004 & 0.76 & $0.14-4.27$ & 0.759 \\
\hline Margin & $36(22)$ & 3.70 & $1.51-9.03$ & 0.004 & 2.39 & $0.57-10.04$ & 0.236 \\
\hline Age & $36(22)$ & 0.99 & $0.91-1.08$ & 0.842 & 0.95 & $0.86-1.05$ & 0.295 \\
\hline
\end{tabular}

Abbreviations: $\mathrm{ASPN}=$ asporin; $\mathrm{BCR}=$ biochemical recurrence; $\mathrm{Cl}=$ confidence interval; $\mathrm{HR}=$ hazards ratio; PSA=prostate specific antigen; TMA = tissue microarray. Cox proportional hazards ratio analysis showed that ASPN mRNA was predictive of BCR in both univariate (hazards ratio 2.16, $P=0.003$ ) and multivariate analysis (hazards ratio 2.23 , $P=0.009$ ), in the Mortensen et al data set derived from microdissected normal and tumour tissue. ASPN mRNA showed a larger hazards ratio than Gleason grade in this data set. Bold indicates statistically significant values.

Table 1B. Hazards ratios of ASPN and BCR; for ASPN mRNA in Mortensen et al, and ASPN H-score in the McGill TMA. Cox analysis of ASPN $\mathrm{H}$-score in the complete McGill TMA

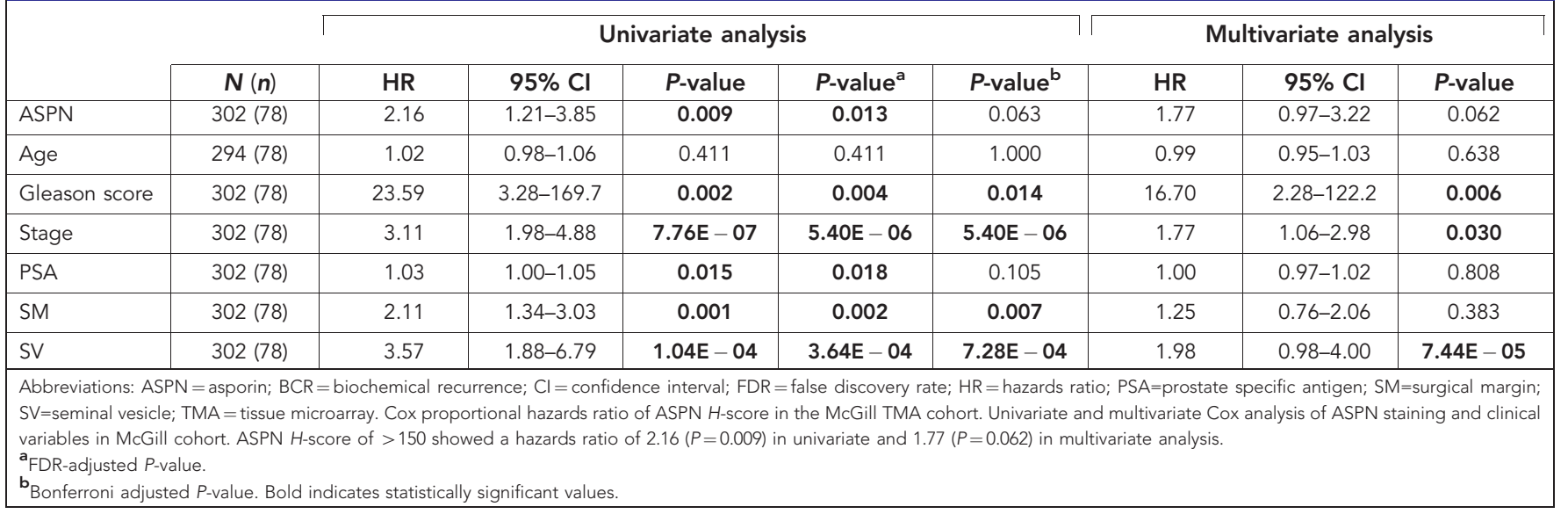

Table 1C. Hazards ratios of ASPN and BCR; for ASPN mRNA in Mortensen et al, and ASPN H-score in the McGill TMA. Cox analysis of ASPN $H$-score in patients with Gleason 7 from the McGill TMA

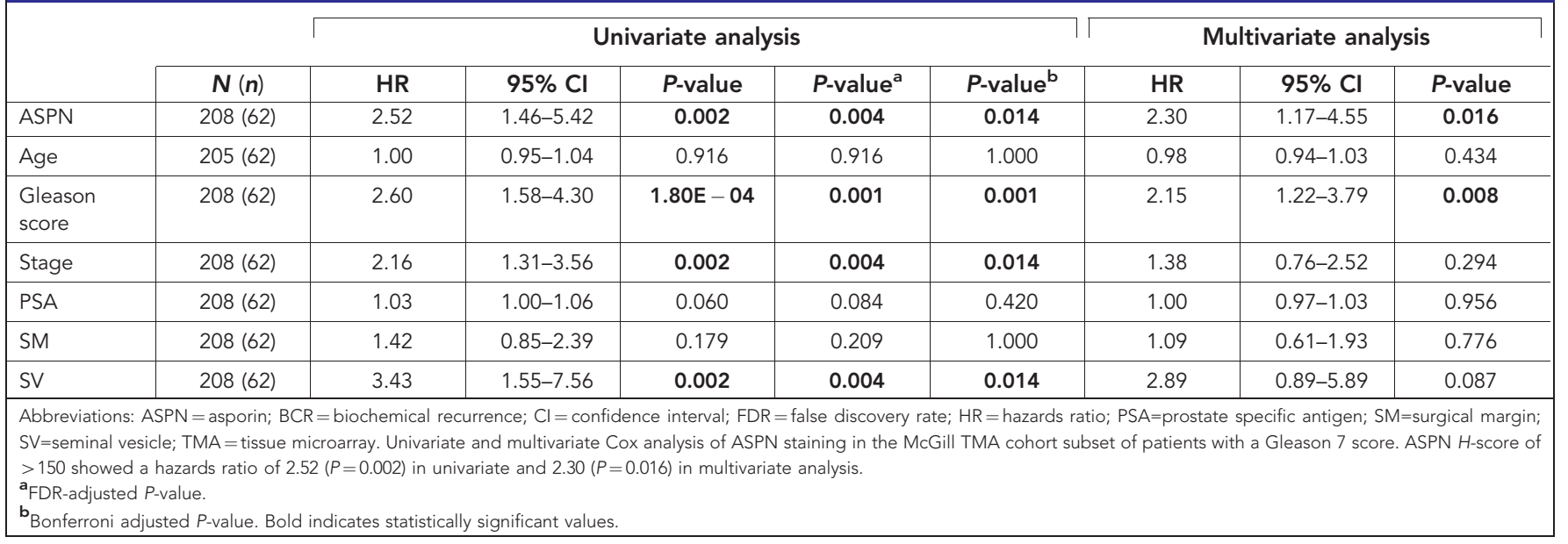

Asporin $H$-score was not associated with BCR in Gleason $3+4$ patients $(P=0.18)$, in contrast to Gleason $4+3$ samples where there was a correlation with $\mathrm{BCR}(P=0.026$; Supplementary Figure $2 \mathrm{~A}$ and $\mathrm{B})$. Similar results from univariate and multivariate analysis using patients with Gleason 6 and Gleason 7 (Supplementary Table S3) show the prognostic potential of ASPN in Gleason sub-group where the need is biggest. The prognostic value of ASPN in Gleason score of 7 was further evident using C-statistics in comparison with pathological Gleason score or the risk group. Risk group was defined by National Comprehensive Cancer Network but here we used Gleason score at surgery instead of biopsy Gleason grade. Low risk patients have a prostate specific antigen (PSA) less than $10 \mathrm{ng} \mathrm{ml}^{-1}$, Gleason score less than 7 and stage less than T2a. Intermediate risk patients have a PSA between 


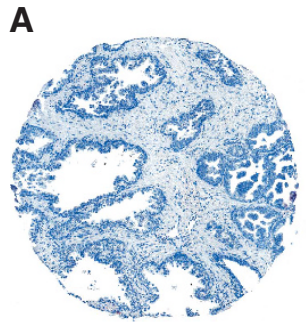

H score: 0

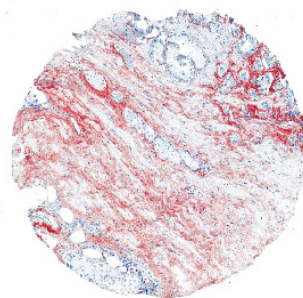

H score: 100
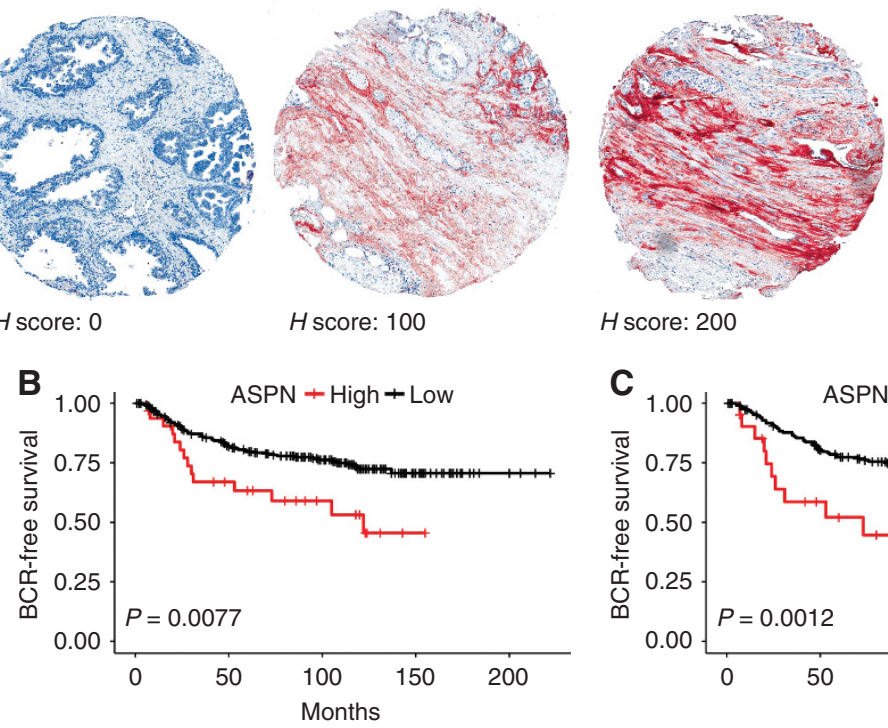

H score: 200

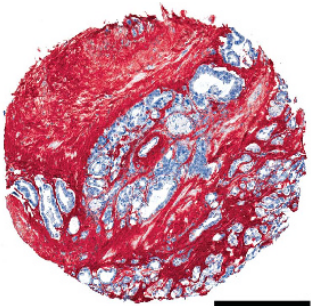

H score: 300

$400 \mu \mathrm{m}$

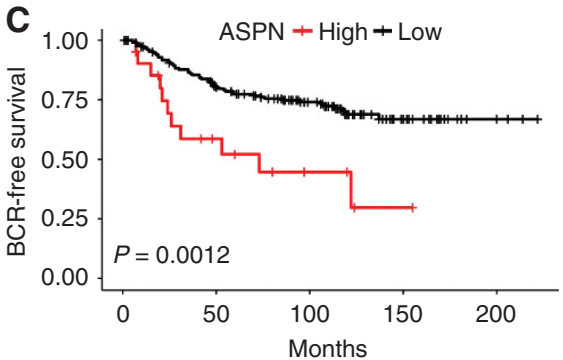

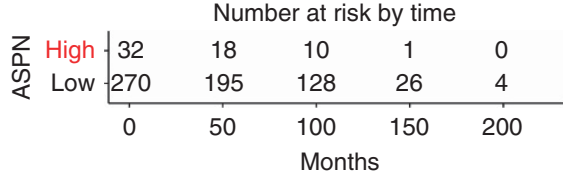

\begin{tabular}{|c|c|c|c|c|c|}
\hline \multirow{4}{*}{$\frac{Z}{\infty}$} & \multicolumn{5}{|c|}{ Number at risk by time } \\
\hline & 21 & 9 & 4 & 1 & 0 \\
\hline & 187 & 138 & 90 & 22 & 4 \\
\hline & 0 & 50 & 100 & 150 & 200 \\
\hline
\end{tabular}

D

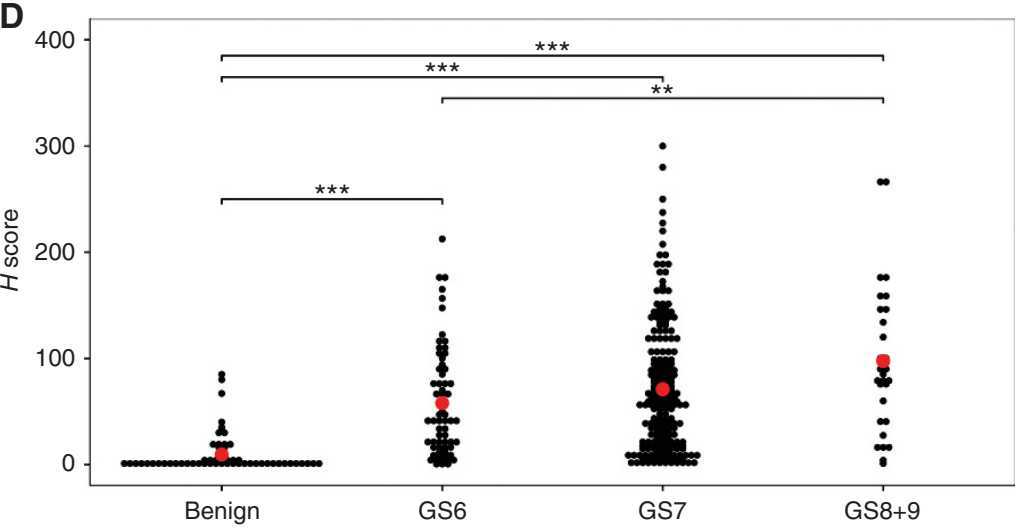

Figure 2. Correlation of ASPN protein expression with BCR using IHC staining of a TMA with patient outcome data. Immunohistochemistry staining of ASPN was undertaken on the McGill cohort of 326 patients with follow up; outcome data and clinico-pathological details are listed in Supplementary Table S1. Asporin staining (brown/red) was observed in stroma and showed peri-tumoural distribution with varying intensity and extent. (A) Representative images of ASPN staining quantified by $\mathrm{H}$-score, with examples of $\mathrm{H}$-scores $0,100,200$, and 300. Scale bar is $400 \mu \mathrm{m}$. $\mathrm{H}$-score combines the staining extent and intensity observed, and was scored by two individuals. (B) Kaplan-Meier analysis of ASPN $H$-score demonstrated that patients with an $H$-score over 150 showed increased BCR $(P=0.0077)$. (C) High ASPN $(H$-score $>150)$ was correlated with increased progression (BCR) in the subset of patients with Gleason grade $7(P=0.0012)$ relative to patients with low ASPN $(H$-score $\leqslant 150)$. Panel $D$ : $H$-score values for ASPN staining and Gleason grade. There was low ASPN staining in benign tissue, and increased ASPN staining in Gleason grades 6, 7, and $8+9$ (ANOVA followed by Tukey multiple comparison, ${ }^{*} P<0.05,{ }^{\star \star} P<0.01$ and ${ }^{\star \star \star} P<0.001$ ). Mean ASPN levels in benign $\mathrm{H}$-score $=9.5$, Gleason $6 \mathrm{H}$-score $=55.9$, Gleason $7 \mathrm{H}$-score $=71.0$, Gleason $8+9 \mathrm{H}$-score $=97.4$.

10 and $20 \mathrm{ng} \mathrm{ml}^{-1}$ and/or Gleason score of 7 and/or a T-stage T2b or T2c. High risk patients have a PSA greater than $20 \mathrm{ng} \mathrm{ml}^{-1}$ and/ or Gleason score between 8 and 10 and/or a T3 stage. ASPN performed better (C-index $0.74, P=3.44 \mathrm{E}^{-04}$ ) than either risk group alone (C-index $0.68, P=0.002)$ or Gleason score alone (C-index 0.72 , $\left.P=2.36 \mathrm{E}^{-05}\right)$. Whereas ASPN improved prognostic capability when combined with either risk group (C-index $0.70, P=2.53 \mathrm{E}^{-05}$ ) or Gleason score (C-index 0.72, $P=1.33 \mathrm{E}^{-06}$; Supplementary Table S2A). Furthermore, multivariate Cox proportional hazard analysis showed that ASPN together with risk group had statistically significant improved prognostic ability over using risk group alone $(P=0.010)$. ASPN together with Gleason score also improved prognostic ability over Gleason score alone $(P=0.021$;
Supplementary Table S2B). These finding demonstrate the independent prognostic value of ASPN in patients with a Gleason score of 7 . In the complete McGill cohort ASPN had also a good prognostic value (C-index $0.68, P=0.007$ ) as well as risk group (C-index 0.76, $P=1.04 \mathrm{E}-13$ ) and Gleason score (C-index 0.97, $P=8.21 \mathrm{E}-45)$. But ASPN did not improve prognostic capability when combined with either risk group (C-index $0.75, P=4.98 \mathrm{E}-15)$ or Gleason score (C-index 0.84, $P=3.63 \mathrm{E}-27$ ) (Supplementary Table S2C). Cox proportional hazard modelling of ASPN H-score in the complete McGill TMA showed a significant association between ASPN and Gleason Grade (Supplementary Table S2D). To examine a correlation between ASPN $H$-score and multiple Gleason grades, we plotted $H$-scores against Gleason 6, 7, and 
$8+9$ combined (Figure 2D). This showed that higher Gleason scores showed statistically significant increased ASPN expression (benign 9.5, Gleason 6 55.8, Gleason 7 71.0, and Gleason $8+9$ 97.4).

Hurley et al (2015) observed association of ASPN with metastatic disease in some but not all prostate cancer cohorts. A comparison of ASPN and the presence of metastatic disease in the McGill cohort showed no significant correlation (Supplementary Figure 2C) though the number of patients with metastatic disease was low. Consequently, we used metastatic samples in two commercially sourced TMAs to examine association of ASPN with metastasis. These TMAs consisted of 24 cores ( 6 patients) and 26 cores (13 patients), where 12 patients had distant metastasis defined by their TNM stage. Staining intensity was examined and scored on a scale of $0-3$. There was low staining observed in cores from a patient with $\mathrm{T} 2 \mathrm{~N} 0 \mathrm{M} 0$ tumour stage when compared to strong staining observed in a patient with T3N0M1b tumour stage (Supplementary Figure 3). Within this small number of patients (12), a comparison of all cores showed a more intense staining for patients with metastasis than those without (Supplementary Figure 3), in contrast to the lack of association in our McGill cohort (Supplementary Figure 2C).

ASPN and reactive stroma. Since we, and others, had observed a correlation of ASPN mRNA and protein with BCR we chose to examine whether ASPN expression might correlate with reactive stroma. Reactive stroma has been associated with progression and prostate cancer specific mortality (Ayala et al, 2003; Yanagisawa et al, 2007) and has been defined using Masson trichrome staining followed by assignation to reactive stroma grade (RSG) $0,1,2$, and 3. RSG0 and 3 are associated with poor outcome, and we speculated that ASPN might be a marker of reactive stroma. To test whether ASPN was associated with reactive stroma, we stained the McGill TMA with Masson's trichrome, and identified samples with low or high levels of reactive stroma (RSG1 + 2 vs RSG0 + 3). These samples had been $\mathrm{H}$-scored for ASPN and thus we could correlate this with the presence or absence of reactive stroma. Trichrome staining and examples of RSG $0,1,2$, and 3 are shown in Figure 3A (high-magnification images are shown in Supplementary Figure S4) and were defined using the criteria described in (Ayala et al, 2003; Yanagisawa et al, 2007). RSG1 and 2 were combined, grouping together samples with $<50 \%$ of the stroma positive for reactive stroma, and defined as low RSG. RSG0 samples were defined as having a very low stromal content, because of high tumour cellularity, where the little stroma present was reactive (ie 100\% reactive stroma, but small stromal proportion). RSG0 was combined with RSG3, comprised of samples with high stromal content and $>50 \%$ reactive stroma-thus the combination of samples with RSG0 and RSG3 were defined as high reactive stroma. We observed that samples with a high reactive stromal
A

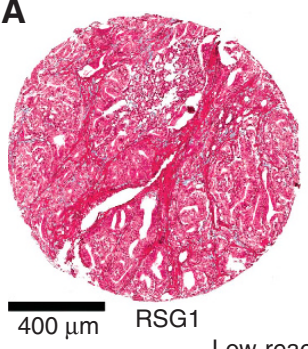

Low-reactive stroma
B

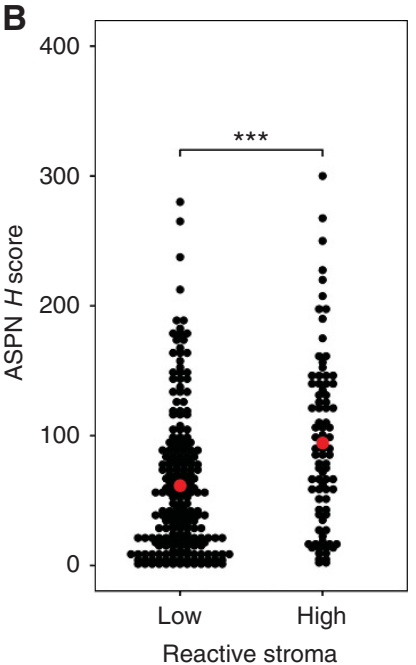

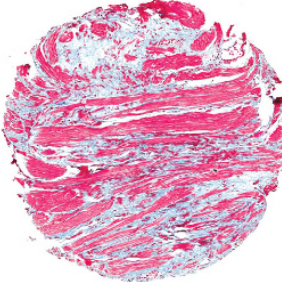

RSG2

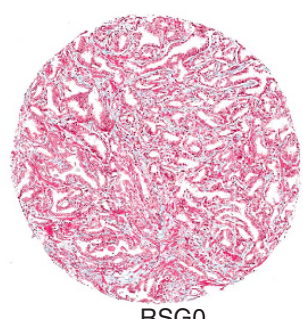

RSGO

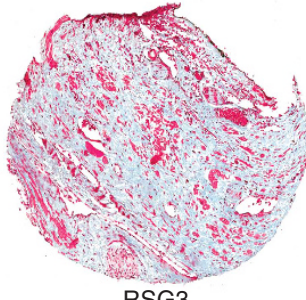

RSG3
C

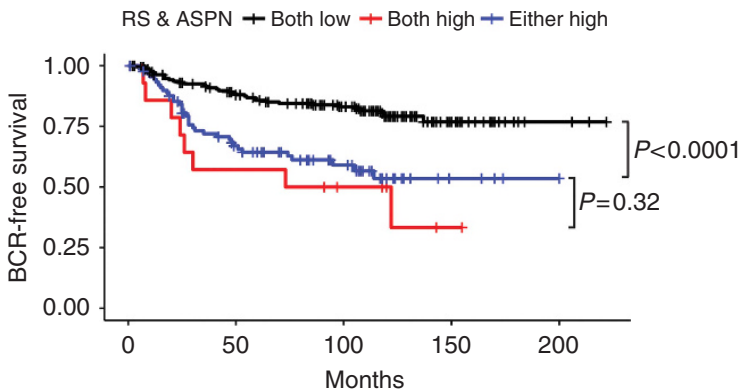

\begin{tabular}{|c|c|c|c|c|c|}
\hline \multirow{5}{*}{$\begin{array}{l}\text { Both low } \\
\text { Both high } \\
\text { Either high }\end{array}$} & \multicolumn{5}{|c|}{ Number at risk by time } \\
\hline & 197 & 154 & 106 & 21 & 3 \\
\hline & 14 & 8 & 5 & 1 & 0 \\
\hline & 91 & 51 & 27 & 5 & 1 \\
\hline & 0 & 50 & 100 & 150 & 200 \\
\hline
\end{tabular}

Figure 3. Co-identification of ASPN and reactive stromal histology. After IHC staining with ASPN and image storage, we stripped the TMA and performed Masson's trichrome staining to identify areas of reactive stroma. Low reactive stroma was scored as RSG1 and 2 while high reactive stroma was RSG0 and RSG3 (Ayala et al, 2003; Yanagisawa et al, 2007). (A) Representative images of samples graded as reactive stroma 1, 2, (low) and 0 , and 3 (high) using Masson's trichrome. (B) Graph of patients showing ASPN staining and reactive stroma; ASPN staining was observed in patients with or without reactive stroma and there was evidence of elevated ASPN staining ( $H$-score) and high reactive stroma (Low reactive stroma samples had a mean $\mathrm{H}$-score of 61.5 while high reactive stroma samples showed an ASPN $H$-score of 94.3, $t$-test $P=0.001$ ). (C) Kaplan-Meier curve of progression among patients with no reactive stroma and low ASPN H-score (black), high reactive stroma and high ASPN (red), and patients showing either reactive stroma or ASPN (blue). Comparison among the three groups showed that samples positive for both reactive stroma and ASPN was predictive of progression as well as samples showing either high reactive stroma or ASPN $(P<0.0001)$. There was no statistically significant difference between the double positive and single positive samples $(P=0.32)$. 
score also showed elevated ASPN $H$-scores (mean $H$-score 94.3), when compared to low reactive stromal samples (mean $H$-score $61.5, P=0.001$ ) (Figure 3B). However, there were clearly some low reactive stroma samples with high levels of ASPN staining, as well as the converse. To investigate the relevance of reactive stroma and ASPN to disease progression, we performed Kaplan-Meier analysis of RSG and ASPN. We observed that patients with low ASPN and low RSG fared better than those with high ASPN and high RSG $(P<0.0001)$. This analysis also showed that patients that were either ASPN positive or RSG positive progressed more rapidly than those with low RSG/ASPN, and were not significantly different from those patients with both reactive stroma and high ASPN $\mathrm{H}$ score (Figure 3C). Individual samples showing the range of ASPN and reactive stroma are shown in Supplementary Figure 5.

Expression of ASPN in stroma of mouse p53 Null model prostate tumours. The correlation between stromal expression of ASPN and progression suggested that expression of ASPN within the stroma may be a response to aggressive tumour types or subclones. To investigate this further, we examined ASPN expression using another antibody (antibody validation Supplementary Figure S6) in a model of aggressive prostate cancer induced by deletion of TP53 in prostate epithelia (Zhou et al, 2006, 2007); to determine whether ASPN was present in tumour cells and/or tumour stroma. Tumours in this model system showed very low stromal content, however we observed ASPN staining within the tumour stroma that was present (Figure 4A-D). The patchy distribution of ASPN staining was reminiscent of that observed in patient tumours (Figure 2A) as well as showing similarity to stroma with RSG0 (Figure 3A). This mouse model often develops neuroendocrine prostate cancer, staining against synaptophysin confirmed that (Figure $4 \mathrm{E}$ and F) suggesting that ASPN is associated with aggressive prostate cancer.

Regulation of ASPN mRNA in Fibroblasts by tumour cell conditioned media. To examine the mechanisms involved in the increased expression of ASPN in peri-tumoural stroma, we tested the effects of cancer cell conditioned media upon the expression of ASPN mRNA in prostate fibroblasts. Our hypothesis was that ASPN might be upregulated by factors secreted from tumour cells, as observed in gastric cancer (Satoyoshi et al, 2014). We prepared serum-free conditioned media from two different prostate cancer cell lines (PC-3 and LNCaP) and added these to fetal prostate fibroblasts (EPF), primary normal prostate stroma (PrSC), and three CAF isolates. After $24 \mathrm{~h}$ exposure to the serum-free conditioned medium stromal cells were collected and ASPN transcript levels measured by qRT-PCR (Figure 5). Normal fibroblasts, both embryonic (EPF) and adult (PrSC), showed an increase of ASPN mRNA when grown with conditioned media from PC-3 cells while conditioned media from LNCaP did not induce $A S P N$ mRNA after $24 \mathrm{~h}$ (Figure 5). CAF derived from three different patients were used and two of these showed increased ASPN transcript levels in response to PC-3 CM. LNCaP CM showed little or no effect and we propose that PC-3 cells express factors that may mimic aggressive tumour subtypes. A cell proliferation assay showed no difference in growth rate between fibroblasts grown with or without CM (data not shown), suggesting that effects upon ASPN levels were not due to changes in proliferation.
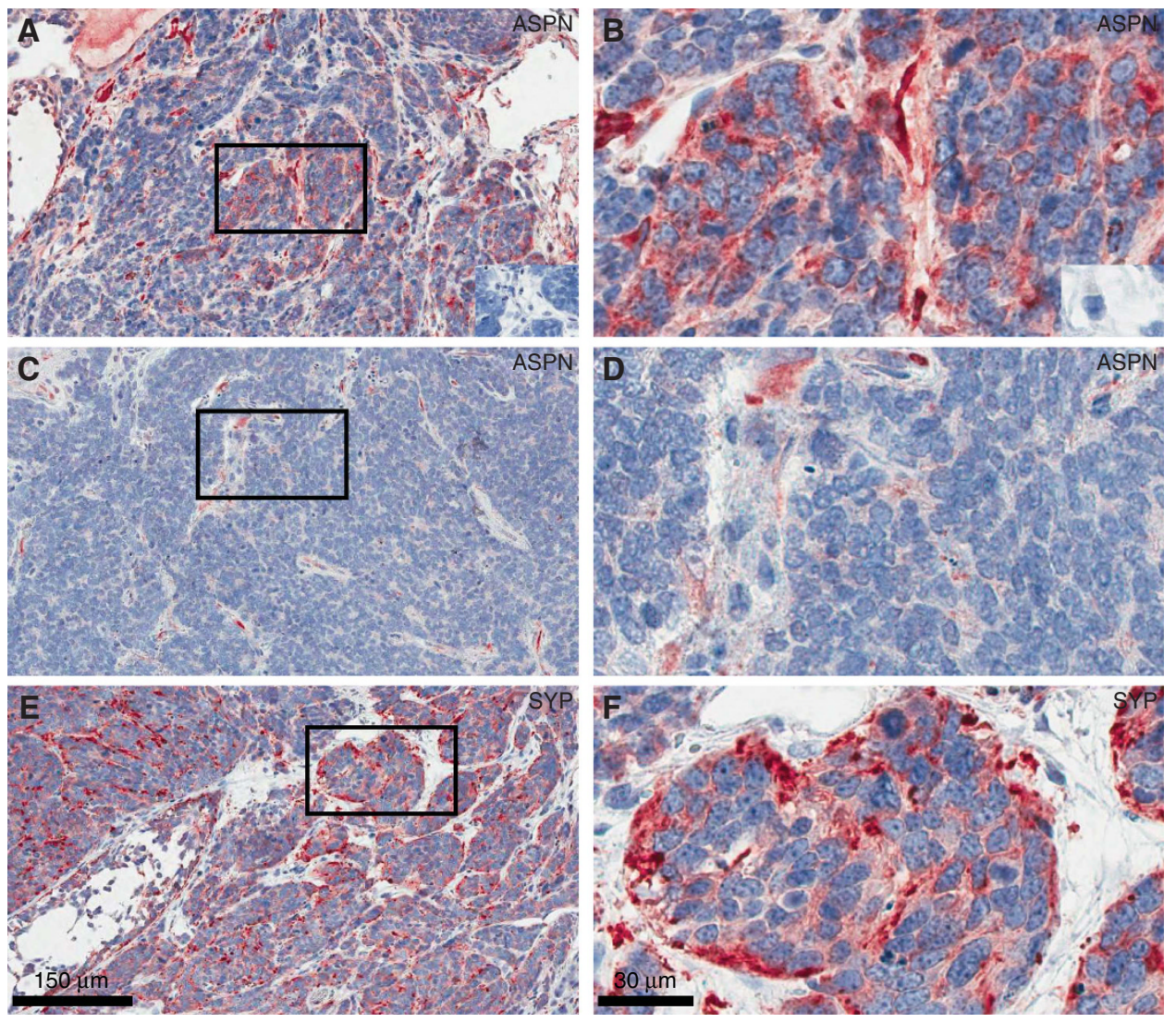

Figure 4. Expression of ASPN in the murine TP53 prostate cancer model. Asporin was localised by immunohistochemistry in the mouse probasin driven TP53 prostate cancer model to determine whether there was expression in stromal cells and/or tumour cells. Prostate tumours from prostate specific p53 knockout mice showed ASPN staining in the stromal compartment (low magnification, A and C; high magnification, B and D). Tumours showed few stromal cells, though many of those present were positive for ASPN. IHC controls without primary antibody are insert in $\mathbf{A}$ and $\mathbf{B}$. Left panels $\times 10$ magnification and right panels at $\times 40$ magnification. Synaptophysin staining is shown in $\mathbf{E}$ and $\mathbf{F}$. 

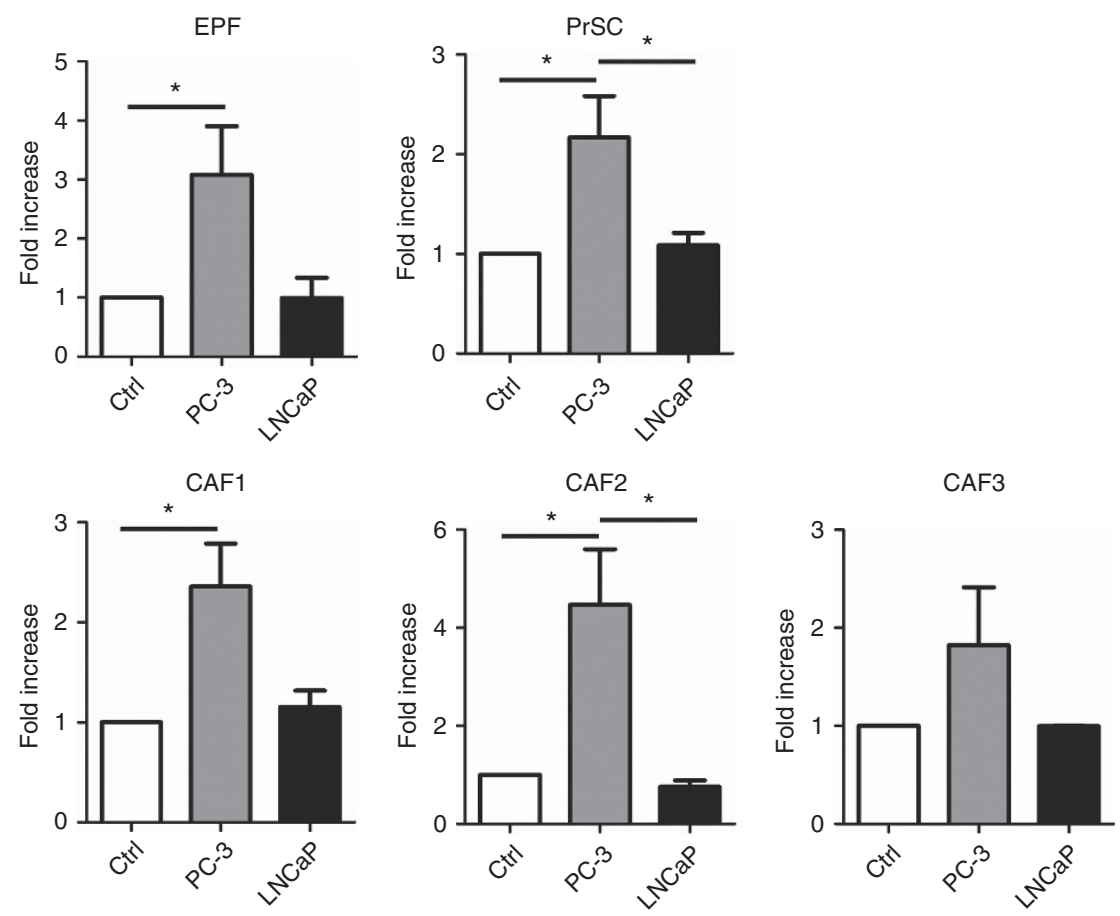

Figure 5. The effect of conditioned media from prostate cancer cell lines upon ASPN expression levels in fibroblasts. Serum-free conditioned media was prepared from PC-3 and LNCaP cells, and added to fetal prostate fibroblasts (EPF), primary prostate stromal cells (PrSC), and 3 isolates of CAFs for $24 \mathrm{~h}$ followed by qRT-PCR analysis of ASPN mRNA. Fold increase compared to control media, and normalised to TBP internal control mRNA. Error bars indicate s.e.m. CM from PC-3 cells induced an increase of ASPN mRNA but LNCaP CM did not; in EPF, PrSC and 2 of 3 CAFs. (ANOVA followed by Tukey multiple comparison, ${ }^{\star} P<0.05$ ).

\section{DISCUSSION}

We show that increased levels of ASPN mRNA or protein are significantly associated with biochemical recurrence in prostate cancer patients following radical prostatectomy, in the McGill cohort and the Mortensen et al (2015) cohort. While the Mortensen cohort was small (36 patients), we suggest that the use of microdissected tissues is important for markers expressed in stroma, as well as validation in independent data sets. KaplanMeier analysis revealed a significant association of ASPN with BCR. Univariate and multivariate analysis of stromal ASPN expression showed ASPN as an independent prognostic factor, and ASPN protein expression was associated with increasing Gleason grade. Our results agree with others that have observed an association of ASPN with prostate cancer (Hurley et al, 2015) and this concordance supports the general conclusion that stromal expression of ASPN is a predictive feature of aggressive prostate cancer subtypes. Hurley et al (2015) also noted an association of ASPN with metastatic recurrence in Mayo Clinic cohort. We were unable to address this in the McGill cohort due to a low rate (5\%, 15 cases) of metastasis, however we did observe an association of ASPN with metastasis in a small commercially sourced TMAs. Hurley et al (2015) also noted that the length of aspartic acid (D)repeat domain in ASPN gene was associated with patient outcome. Germline ASPN D13/14 (or any D14) was significantly associated with metastatic recurrence following surgery while ASPN D13/13 was associated with a reduced risk of metastatic progression. Germline ASPN D13/14 was also associated with biochemical recurrence following surgery but when controlled for pre-surgical variables in multivariable Cox regression analysis the hazards ratio was not significant.

The association between ASPN and metastatic recurrence in prostate cancer may be due to increased co-invasion of CAFs and cancer cells (Gaggioli et al, 2007) and as established in gastric cancer. Asporin activates Rac1 via an interaction with CD44 and promotes invasion of CAFs and may also transactivate Rac1 in cancer cells to stimulate their invasion (Satoyoshi et al, 2014). Fibroblasts overexpressing ASPN (D14 variant) mixed with PC-3 cells and then grafted into murine prostates showed a higher number of metastases to distant lymph nodes and other organs including lung, liver and pancreas than control cells (Hurley et al, 2015). It was previously revealed that ASPN regulates BMP2 and TGF $\beta 1$ activity through direct and indirect interactions (Kizawa et al, 2005; Yamada et al, 2007; Tomoeda et al, 2008). Among its roles TGF $\beta$ serves as a tumour suppressor by inducing cell cycle arrest and or apoptosis (Wikstrom et al, 2001). Asporin negatively regulates TGF $\beta$ and this leads to an increase in cell proliferation and a decrease in apoptosis. More recently it has been shown that ASPN regulates TLR2- and TLR4-induced inflammatory responses (Yamaba et al, 2015) and also positively regulates FGF2 activity (Awata et al, 2015). FGF2 is increased in prostate cancer compared to normal prostate tissue, and increased FGF2 is localised to the tumour stroma. FGF2 may induce matrix metalloproteinases (Miyake et al, 1997; Linder et al, 1998) which could enhance tissue invasion by the tumour cells in vivo.

It is now well accepted that the tumour microenvironment plays an important role in tumour progression (Quail and Joyce, 2013). Recent studies have identified other stromal markers associated with prostate cancer progression. Patients with high expression of VEGF-A in stroma and/or high expression of VEGFR-2 in stroma had higher rates of biochemical failure. Patients with high stromal expression of VEGFR-2 had a significantly worse outcome regarding clinical failure defined as a palpable tumour recurrence in the prostate bed or metastasis (Nordby et al, 2015). Similarly, strong MCT4 density in stroma was a significant prognosticator of lower biochemical failure-free survival. However in multivariate analysis MTC4 expression alone had no individual independent prognostic effect on BCR when adjusting for acknowledged and significant clinicopathological variables. But coexpression of MTC1 
in tumour and MCT4 in stroma was independently and significantly associated with BCR (Andersen et al, 2015). Periostin, an extracellular matrix protein has been reported to be able to distinguish prostate cancer from $\mathrm{BPH}$ and PIN and correlates with increasing Gleason score (Tian et al, 2015). Taken together, it appears that stromal markers are correlated with BCR, but that better performing markers are required for patient stratification and treatment choice.

In gastric cancer, expression of ASPN in fibroblasts was increased upon incubation of the cells in conditioned medium from gastric epithelial cells (Satoyoshi et al, 2014). We observed a similar increase in expression of ASPN mRNA in prostate fibroblasts following incubation with conditioned media with the prostate cancer PC-3 cell line. Normal fibroblasts showed increased levels of ASPN but this was only observed in 2 out of 3 CAF lines. This may reflect CAF heterogeneity, which is well documented. We observed that $\mathrm{CM}$ from LNCaP cells was unable to induce ASPN mRNA levels suggesting that there may be PC-3 or tumour specific regulators of stromal ASPN expression. It supports the hypothesis that expression of ASPN in tumour stroma is a response to aggressive tumour subtypes. This is further supported by our observation of Aspn expression in the stroma of the TP53 mouse model of prostate cancer.

In conclusion, we demonstrated that ASPN expression is correlated with disease progression in prostate cancer using a large cohort, consistent with recent studies. Experimentally, we observed expression of Aspn in stroma in a mouse prostate cancer model, and we demonstrated that cancer cell lines could increase ASPN expression in fibroblasts. This suggests that ASPN is expressed in response to tumour cell derived factors in patients and that a stromal response to aggressive tumour subclones may be of prognostic value.

\section{ACKNOWLEDGEMENTS}

NB and AAT were supported by a Prostate Cancer Canada Team grant, Grant \#T2014-01, proudly funded by the Movember Foundation.

${ }^{4}$ Current address: Division of Surgery and Interventional Sciences, Lab 2.4, University College London, Cruciform Building, Gower Street, London, WC1E 6BT, UK.

${ }^{5}$ Current address: AstraZeneca, R\&D Oncology iMed, Lab 240, CRUK-Cambridge Institute, Li Ka Shing Centre, Robinson Way, Cambridge CB2 0RE, UK.

${ }^{6}$ Current address: University of Oxford, John Radcliffe Hospital, Headley Way, Headington, Oxford OX3 9DU, UK.

\section{CONFLICT OF INTEREST}

The authors declare no conflict of interest.

\section{REFERENCES}

Andersen S, Solstad O, Moi L, Donnem T, Eilertsen M, Nordby Y, Ness N, Richardsen E, Busund LT, Bremnes RM (2015) Organized metabolic crime in prostate cancer: The coexpression of MCT1 in tumor and MCT4 in stroma is an independent prognosticator for biochemical failure. Urol Oncol 33: 338.e339-317.

Awata T, Yamada S, Tsushima K, Sakashita H, Yamaba S, Kajikawa T, Yamashita M, Takedachi M, Yanagita M, Kitamura M, Murakami S (2015) PLAP-1/Asporin Positively Regulates FGF-2 Activity. J Dent Res 94: $1417-1424$.

Ayala G, Tuxhorn JA, Wheeler TM, Frolov A, Scardino PT, Ohori M, Wheeler M, Spitler J, Rowley DR (2003) Reactive stroma as a predictor of biochemical-free recurrence in prostate cancer. Clin Cancer Res 9: 4792-4801.

Bosman FT, de Goeij AF, Rousch M (1992) Quality control in immunocytochemistry: experiences with the oestrogen receptor assay. J Clin Pathol 45: 120-124.

Dakhova O, Ozen M, Creighton CJ, Li R, Ayala G, Rowley D, Ittmann M (2009) Global gene expression analysis of reactive stroma in prostate cancer. Clin Cancer Res 15: 3979-3989.

Franco OE, Jiang M, Strand DW, Peacock J, Fernandez S, Jackson 2nd RS, Revelo MP, Bhowmick NA, Hayward SW (2011) Altered TGF-beta signaling in a subpopulation of human stromal cells promotes prostatic carcinogenesis. Cancer Res 71: 1272-1281.

Gaggioli C, Hooper S, Hidalgo-Carcedo C, Grosse R, Marshall JF, Harrington K, Sahai E (2007) Fibroblast-led collective invasion of carcinoma cells with differing roles for RhoGTPases in leading and following cells. Nat Cell Biol 9: 1392-1400.

Hurley PJ, Sundi D, Shinder B, Simons BW, Hughes RM, Miller RM, Benzon B, Faraj SF, Netto GJ, Vergara IA, Erho N, Davicioni E, Karnes RJ, Yan G, Ewing C, Isaacs SD, Berman DM, Rider JR, Jordahl KM, Mucci LA, Huang J, An SS, Park BH, Isaacs WB, Marchionni L, Ross AE, Schaeffer EM (2015) Germline variants in asporin vary by race, modulate the tumor microenvironment, and are differentially associated with metastatic prostate cancer. Clin Cancer Res 22(2): 448-458.

Jia Z, Rahmatpanah FB, Chen X, Lernhardt W, Wang Y, Xia XQ, Sawyers A, Sutton M, McClelland M, Mercola D (2012) Expression changes in the stroma of prostate cancer predict subsequent relapse. PLoS ONE 7: e41371.

Jia Z, Wang Y, Sawyers A, Yao H, Rahmatpanah F, Xia XQ, Xu Q, Pio R, Turan T, Koziol JA, Goodison S, Carpenter P, Wang-Rodriguez J, Simoneau A, Meyskens F, Sutton M, Lernhardt W, Beach T, Monforte J, McClelland M, Mercola D (2011) Diagnosis of prostate cancer using differentially expressed genes in stroma. Cancer Res 71: 2476-2487.

Kassambara A (2016) Drawing Survival Curves using 'ggplot2'. Available at: http://CRAN.r-project.org/web/packages/survminer.

Kizawa H, Kou I, Iida A, Sudo A, Miyamoto Y, Fukuda A, Mabuchi A, Kotani A, Kawakami A, Yamamoto S, Uchida A, Nakamura K, Notoya K, Nakamura Y, Ikegawa S (2005) An aspartic acid repeat polymorphism in asporin inhibits chondrogenesis and increases susceptibility to osteoarthritis. Nat Genet 37: 138-144.

Klee EW, Bondar OP, Goodmanson MK, Dyer RB, Erdogan S, Bergstralh EJ, Bergen 3rd HR, Sebo TJ, Klee GG (2012) Candidate serum biomarkers for prostate adenocarcinoma identified by mRNA differences in prostate tissue and verified with protein measurements in tissue and blood. Clin Chem 58: 599-609.

Kou I, Nakajima M, Ikegawa S (2010) Binding characteristics of the osteoarthritisassociated protein asporin. J Bone Miner Metab 28: 395-402.

Lau C, Kim Y, Chia D, Spielmann N, Eibl G, Elashoff D, Wei F, Lin YL, Moro A, Grogan T, Chiang S, Feinstein E, Schafer C, Farrell J, Wong DT (2013) Role of pancreatic cancer-derived exosomes in salivary biomarker development. J Biol Chem 288: 26888-26897.

Linder C, Bystrom P, Engel G, Auer G, Aspenblad U, Strander H, Linder S (1998) Correlation between basic fibroblast growth factor immunostaining of stromal cells and stromelysin-3 mRNA expression in human breast carcinoma. Br J Cancer 77: 941-945.

Maris P, Blomme A, Palacios AP, Costanza B, Bellahcene A, Bianchi E, Gofflot S, Drion P, Trombino GE, Di Valentin E, Cusumano PG, Maweja S, Jerusalem G, Delvenne P, Lifrange E, Castronovo V, Turtoi A (2015) Asporin Is a Fibroblast-Derived TGF-beta1 Inhibitor and a Tumor Suppressor Associated with Good Prognosis in Breast Cancer. PLoS Med 12: e1001871.

Martin NE (2016) New developments in prostate cancer biomarkers. Curr Opin Oncol 28: 248-252.

Miyake H, Yoshimura K, Hara I, Eto H, Arakawa S, Kamidono S (1997) Basic fibroblast growth factor regulates matrix metalloproteinases production and in vitro invasiveness in human bladder cancer cell lines. J Urol 157: 2351-2355.

Mortensen MM, Hoyer S, Lynnerup AS, Orntoft TF, Sorensen KD, Borre M, Dyrskjot L (2015) Expression profiling of prostate cancer tissue delineates genes associated with recurrence after prostatectomy. Sci Rep 5: 16018.

Nordby Y, Andersen S, Richardsen E, Ness N, Al-Saad S, Melbo-Jorgensen C, Patel HR, Donnem T, Busund LT, Bremnes RM (2015) Stromal expression of VEGF-A and VEGFR-2 in prostate tissue is associated with biochemical and clinical recurrence after radical prostatectomy. Prostate 75: $1682-1693$. 
Olumi AF, Grossfeld GD, Hayward SW, Carroll PR, Tlsty TD, Cunha GR (1999) Carcinoma-associated fibroblasts direct tumor progression of initiated human prostatic epithelium. Cancer Res 59: 5002-5011.

Orr B, Riddick AC, Stewart GD, Anderson RA, Franco OE, Hayward SW, Thomson AA (2012) Identification of stromally expressed molecules in the prostate by tag-profiling of cancer-associated fibroblasts, normal fibroblasts and fetal prostate. Oncogene 31: 1130-1142.

Orr B, Vanpoucke G, Grace OC, Smith L, Anderson RA, Riddick AC, Franco OE, Hayward SW, Thomson AA (2011) Expression of pleiotrophin in the prostate is androgen regulated and it functions as an autocrine regulator of mesenchyme and cancer associated fibroblasts and as a paracrine regulator of epithelia. Prostate 71: 305-317.

Pascal LE, Goo YA, Vencio RZ, Page LS, Chambers AA, Liebeskind ES, Takayama TK, True LD, Liu AY (2009) Gene expression down-regulation in $\mathrm{CD} 90+$ prostate tumor-associated stromal cells involves potential organ-specific genes. BMC Cancer 9: 317.

Pena C, Cespedes MV, Lindh MB, Kiflemariam S, Mezheyeuski A, Edqvist PH, Hagglof C, Birgisson H, Bojmar L, Jirstrom K, Sandstrom P, Olsson E, Veerla S, Gallardo A, Sjoblom T, Chang AC, Reddel RR, Mangues R, Augsten M, Ostman A (2013) STC1 expression by cancer-associated fibroblasts drives metastasis of colorectal cancer. Cancer Res 73: 1287-1297.

Planche A, Bacac M, Provero P, Fusco C, Delorenzi M, Stehle JC, Stamenkovic I (2011) Identification of prognostic molecular features in the reactive stroma of human breast and prostate cancer. PLoS ONE 6: e18640.

Quail DF, Joyce JA (2013) Microenvironmental regulation of tumor progression and metastasis. Nat Med 19: 1423-1437.

Satoyoshi R, Kuriyama S, Aiba N, Yashiro M, Tanaka M (2014) Asporin activates coordinated invasion of scirrhous gastric cancer and cancerassociated fibroblasts. Oncogene 34(5): 650-660.

Schaeffer EM, Marchionni L, Huang Z, Simons B, Blackman A, Yu W, Parmigiani G, Berman DM (2008) Androgen-induced programs for prostate epithelial growth and invasion arise in embryogenesis and are reactivated in cancer. Oncogene 27: 7180-7191.

Schroder MS, Culhane AC, Quackenbush J, Haibe-Kains B (2011) survcomp: an $\mathrm{R} /$ Bioconductor package for performance assessment and comparison of survival models. Bioinformatics 27: 3206-3208.

Simkova D, Kharaishvili G, Korinkova G, Ozdian T, Suchankova-Kleplova T, Soukup T, Krupka M, Galandakova A, Dzubak P, Janikova M, Navratil J, Kahounova Z, Soucek K, Bouchal J (2016) The dual role of asporin in breast cancer progression. Oncotarget 7(32): 52045-52060.

Tanner MJ, Welliver Jr RC, Chen M, Shtutman M, Godoy A, Smith G, Mian BM, Buttyan R (2011) Effects of androgen receptor and androgen on gene expression in prostate stromal fibroblasts and paracrine signaling to prostate cancer cells. PloS one 6: e16027.
Team, R.D.C. (2011) R: A Language and Environment for Statistical Computing. Vienna, Austria: the R Foundation for Statistical Computing. ISBN: 3-900051-07-0. Available at http://www.R-project.org/.

Therneau T (2015) A Package for Survival Analysis in S. version 2.38. Available at: http://CRAN.R-project.org/package=survival.

Tian Y, Choi CH, Li QK, Rahmatpanah FB, Chen X, Kim SR, Veltri R, Chia D, Zhang Z, Mercola D, Zhang H (2015) Overexpression of periostin in stroma positively associated with aggressive prostate cancer. PLOS ONE 10: e0121502.

Tomoeda M, Yamada S, Shirai H, Ozawa Y, Yanagita M, Murakami S (2008) PLAP-1/asporin inhibits activation of BMP receptor via its leucine-rich repeat motif. Biochem Biophys Res Commun 371: 191-196.

Wikstrom P, Damber J, Bergh A (2001) Role of transforming growth factorbeta1 in prostate cancer. Microsc Res Tech 52: 411-419.

Yamaba S, Yamada S, Kajikawa T, Awata T, Sakashita H, Tsushima K, Fujihara C, Yanagita M, Murakami S (2015) PLAP-1/Asporin Regulates TLR2- and TLR4-induced Inflammatory Responses. J Dent Res 94(12): 1706-1714.

Yamada S, Tomoeda M, Ozawa Y, Yoneda S, Terashima Y, Ikezawa K, Ikegawa S, Saito M, Toyosawa S, Murakami S (2007) PLAP-1/asporin, a novel negative regulator of periodontal ligament mineralization. J Biol Chem 282: 23070-23080.

Yanagisawa N, Li R, Rowley D, Liu H, Kadmon D, Miles BJ, Wheeler TM, Ayala GE (2007) Stromogenic prostatic carcinoma pattern (carcinomas with reactive stromal grade 3 ) in needle biopsies predicts biochemical recurrence-free survival in patients after radical prostatectomy. Hum Pathol 38: 1611-1620.

Zhou Z, Flesken-Nikitin A, Corney DC, Wang W, Goodrich DW, Roy-Burman P, Nikitin AY (2006) Synergy of p53 and Rb deficiency in a conditional mouse model for metastatic prostate cancer. Cancer Res 66: 7889-7898.

Zhou Z, Flesken-Nikitin A, Nikitin AY (2007) Prostate cancer associated with p53 and Rb deficiency arises from the stem/progenitor cellenriched proximal region of prostatic ducts. Cancer Res 67: 5683-5690.

(c) (i) (2) This work is licensed under the Creative Commons (c) ${ }_{\text {BY }}{ }_{\text {NC }}$ AA Attribution-Non-Commercial-Share Alike 4.0 International License. To view a copy of this license, visit http:// creativecommons.org/licenses/by-nc-sa/4.0/

(C) The Author(s) named above 2017

Supplementary Information accompanies this paper on British Journal of Cancer website (http://www.nature.com/bjc) 